\title{
Testing ATHENA optics: a new measurement standard at the PANTER x-ray test facility
}

Miranda Bradshaw, Vadim Burwitz, Gisela Hartner, Andreas Langmeier, Giuseppe Vacanti, et al.

Miranda Bradshaw, Vadim Burwitz, Gisela Hartner, Andreas Langmeier, Giuseppe Vacanti, Maximilien J. Collon, Nicolas M. Barrière, "Testing ATHENA optics: a new measurement standard at the PANTER $x$-ray test facility," Proc. SPIE 11852, International Conference on Space Optics ICSO 2020, 1185223 (11 June 2021); doi: 10.1117/12.2599664

SPIE Event: International Conference on Space Optics - ICSO 2021, 2021, Online Only 


\section{International Conference on Space Optics-ICSO 2020}

Virtual Conference

30 March-2 April 2021

Edited by Bruno Cugny, Zoran Sodnik, and Nikos Karafolas
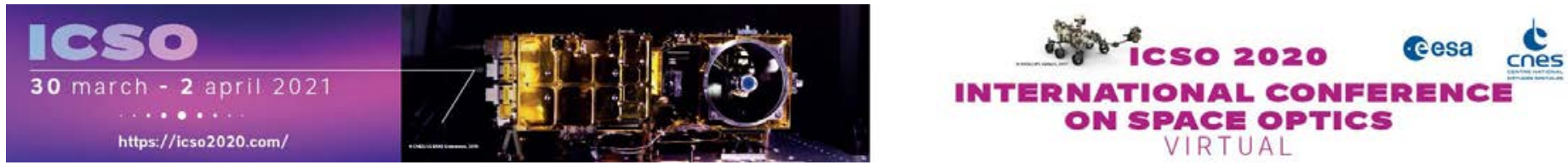

\section{Testing ATHENA optics: a new measurement standard at the PANTER $x$-ray test facility}

\section{Cesa issopoceatings lecnes}




\title{
Testing ATHENA Optics - A New Measurement Standard at the PANTER X-Ray Test Facility
}

\author{
Miranda Bradshaw*1, Vadim Burwitz ${ }^{1}$, Gisela Hartner ${ }^{1}$, Andreas Langmeier ${ }^{1}$, Giuseppe Vacanti ${ }^{2}$, \\ Maximilien J. Collon², Nicolas M. Barrière ${ }^{2}$ \\ ${ }^{1}$ Max Planck Institute for Extraterrestrial Physics, Giessenbachstrasse 1, 85748 Garching, Germany \\ ${ }^{2}$ cosine measurement systems, Oosteinde 36, 2361 HE Warmond, The Netherlands \\ *bradshaw@mpe.mpg.de
}

\begin{abstract}
The European Space Agency ATHENA mission is an x-ray observatory that will study the formation of galaxy clusters and the growth of black holes. Due for launch in early 2030s, with adoption planned for 2022, ATHENA will use 600 silicon pore optic mirror modules to create a $2.6 \mathrm{~m}$ diameter $\mathrm{x}$-ray mirror.

The PANTER $\mathrm{x}$-ray test facility has over 40 years of experience in testing and calibrating $\mathrm{x}$-ray optics. PANTER plays a key part in testing developments of silicon pore optics, manufactured by cosine measurement systems, for the ATHENA mission.

In 2020, as part of these developments, an x-ray optic unit comprising two mandrels manufactured by Zeiss was measured at PANTER. This optic was produced as a standard against which the performance of silicon pore optics and of the facilities involved in the fabrication and testing of silicon pore optics can be measured.

The paper will provide an overview of the testing carried out at PANTER, and the performance of the optic.

Keywords: $\mathrm{x}$-ray optics, $\mathrm{x}$-ray testing, ATHENA, silicon pore optics, $\mathrm{x}$-ray astronomy

\section{THE OBJECTIVE}

The objective of the measurement campaign was to determine the performance of the optic, specifically the half-energy width (HEW) at $1.49 \mathrm{keV}(\mathrm{Al}-\mathrm{K})$. This optic is to serve as the optical 'standard' to compare the $\mathrm{x}$-ray measurement capabilities of different facilities, in preparation for the ATHENA mirror construction and testing.
\end{abstract}

\section{THE OPTIC}

The optic tested in this paper was built by cosine measurement systems, and is identified as XOU-0055 (MM-0037). XOU0055 is a single-shell system, comprising two mandrels from Zeiss - one characterised to represent the parabolic mirror and the other the hyperbolic mirror. Together the two mandrels form a single reflecting 'layer' of the x-ray optic unit (XOU). General information on the manufacturing and construction of silicon pore optics can be found elsewhere ${ }^{1,2}$. XOU-0055 was mounted inside a mirror module (MM) structure for testing at PANTER. A dummy block was placed in the second XOU position of the MM, to give the necessary mechanical stiffness to the optic structure. The dummy block did not contribute to the measurement results.

Table 2-1 gives the specifications of the optic. Figure 2-1 shows a photograph of the optic prior to installation at the PANTER facility. 
Table 2-1: Specifications of the XOU-0055 optic, comprising two mandrels to create one silicon reflective 'layer'

\begin{tabular}{|l|c|}
\hline Parameter & Value \\
\hline MM name & MM-0037 \\
\hline XOU name & XOU-0055 \\
\hline Number of silicon pore layers & 1 \\
\hline Radius of curvature & $737 \mathrm{~mm}$ \\
\hline Focal length (nominal) & $12000 \mathrm{~mm}$ \\
\hline Focal length (measured at BESSY) & $11975 \mathrm{~mm}$ \\
\hline Separation between mandrels & $0.5 \pm 0.2 \mathrm{~mm}$ \\
\hline
\end{tabular}

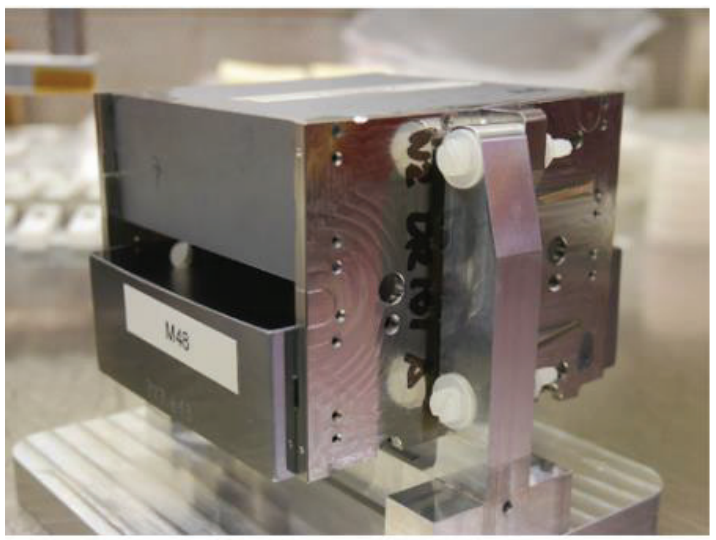

(a)

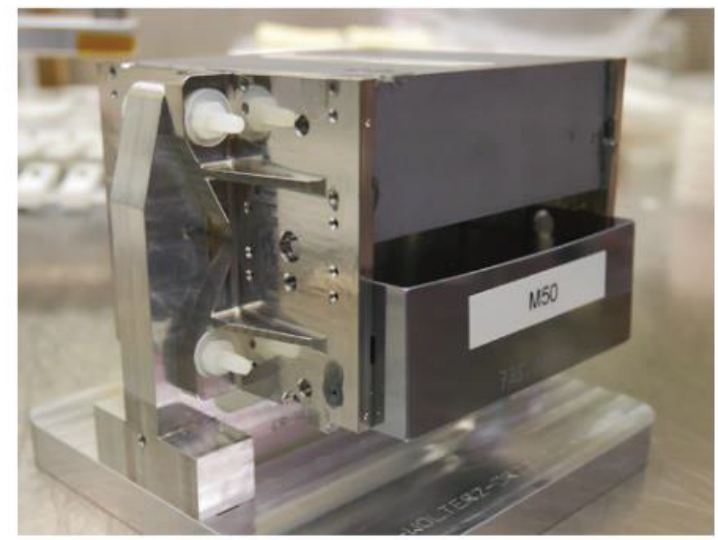

(b)

Figure 2-1: Photograph of the XOU-0055 mandrel optic, showing (a) the parabolic M48 mandrel and (b) the hyperbolic M50 mandrel (Photo credit: cosine measurement systems)

\section{TEST METHODOLOGY}

The test method for this optic followed the same method as previous ATHENA MM test campaigns at PANTER ${ }^{4,6}$. As such, only a brief description is given here.

\subsection{Installation and Setup}

The optic was installed on its side, as the $\sim 12 \mathrm{~m}$ focal length of the optic restricted the setup to certain configurations. Figure 3-1a shows the optic installed in the PANTER chamber attached to a hexapod, which allows fine movements of the optic once under vacuum. The pivot point of the hexapod is set to the centre of the optic. To assist the reader in visualising the measurements in this paper, the 'top' of the optic is the edge of the optic closest to the roof of the facility, and the 'bottom' of the optic is closest to the ground. This coordinate system is indicated in Figure 3-1b.

A moveable mask was installed between the optic and x-ray source, with apertures capable of illuminating 100\%, 48\%, and $4 \%$ of the optic. These apertures were chosen to enable characterisation of the full optic and of specific sections of the optic. The mask is visible in Figure 3-1a. 


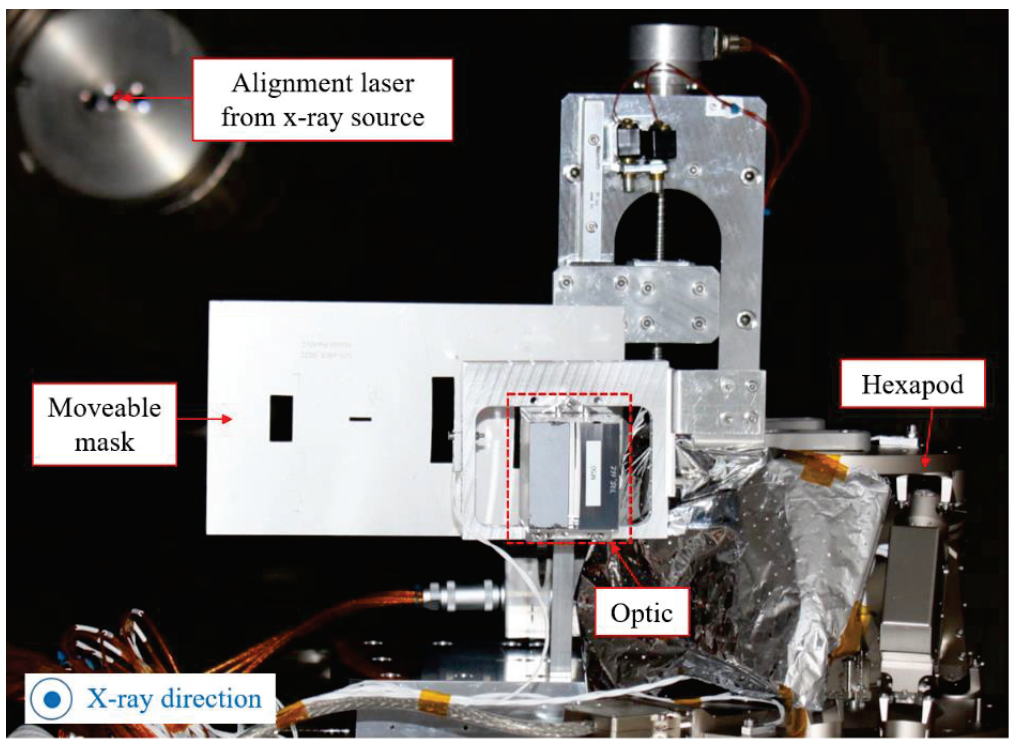

(a)

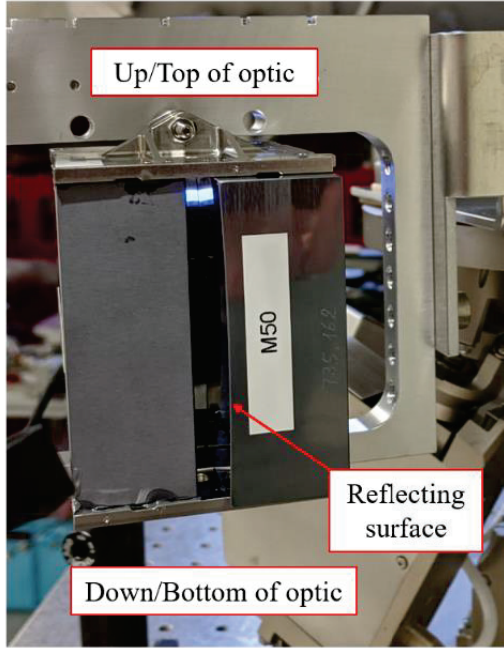

(b)

Figure 3-1: (a) The XOU-0055 mounted inside the PANTER chamber attached to a hexapod, to enable fine adjustment of the optic under vacuum. The moveable mask used to illuminate either the full optic or a chosen section of the optic is also shown. The MLI between the optic and the hexapod protects the optic from the heat produced by the hexapod motors. (b) The optic in its holding structure at the PANTER facility, showing the nomenclature for the measurements of the optic during this campaign. (Credit: MPE)

\subsection{Alignment}

Once under x-ray illumination, the $100 \%$ mask was aligned over the optic by observing the total x-ray count rate for the optic. The mask was moved incrementally and the corresponding change in count rate was observed using the online detector tool. Once the 100\% mask was aligned, the 4\% mask was aligned using geometric distances from the mask CAD drawing, then finely adjusted by eye to observe when the x-ray scattering around the point spread function (PSF) was and was not visible.

The optic was aligned in pitch and yaw using the $100 \%$ mask. This is part of the standard optical alignment method at PANTER, the aim of which is to minimise the HEW and maximise the flux over a range of pitch and yaw angles ${ }^{4}$.

Once the optic alignment in pitch and yaw was completed, a focus search ${ }^{4}$ was carried out to determine the best focus position for the detector. The detector was translated towards and away from the $\mathrm{x}$-ray source $\pm 50 \mathrm{~mm}$ around the initial in-chamber alignment position in steps of $5 \mathrm{~mm}$. An exposure was made at each of these positions, and the corresponding HEW, x-FWHM, and y-FWHM calculated. Fitting a parabola to the data determined the minimum HEW and y-FWHM and the corresponding 'best focus' position. The y-FWHM was taken as the final fitting metric, due to the variance of the HEW across the optic surface.

Cosine had measured the optic at BESSY, at the PTB XPBF 2.0 beamline $^{7}$, prior to this campaign. As such, it was known that the best focus position varied across the optic. It was decided that a second focus search should be carried out, for the bottom half of the optic, using the $48 \%$ mask, where the best HEW performance was expected. The bottom-of-optic best focus position deviated from the complete optic best focus position by $28.7 \mathrm{~mm}$. 


\subsection{Measurements}

All measurements were made at Al-K $(1.49 \mathrm{keV})$.

\subsubsection{Single-Exposure PSF (HEW)}

Single-exposure PSF images were made at the position of best focus for the full optic, using the $100 \%$ mask, for approximately 10,000 counts. These exposures were used as preliminary HEW measurements, prior to the pixel scans (Section 3.3.3) being made.

\subsubsection{Azimuthal Scan (HEW)}

An azimuthal scan was carried out using the 4\% mask in the best focus position for the full optic. This type of scan allows closer analysis of the optic surface, as the $4 \%$ mask illuminates only $2.5 \mathrm{~mm}$ of the optic per exposure. A total of 24 exposures were made, starting from the top of the optic and covering the full length of the optic.

\subsubsection{Pixel Scan (HEW)}

Following the single PSF images and the azimuthal scan, two pixel scans ${ }^{3,5}$ were made: one for the full optic and one for the bottom of the optic. A pixel scan increases the measurement resolution of the HEW by enabling sub-pixel resolution; the detector pixels are $0.075 \mathrm{~mm} \times 0.075 \mathrm{~mm}$, and with a 6x6-exposure pixel scan the detector movement pitch is 0.0125 $\mathrm{mm}$. This measurement scans over the pixel, and is necessary because the PSF of this optic is so small that the photons encircled in the "HEW circle" during the analysis are mainly detected in one pixel. Each full pixel scan (36 exposures) yields one HEW value.

\subsubsection{Intra-Focal/Extra-Focal Images}

Intra-focal and extra-focal images were made of the optic, to expose any features that were not visible in the focused PSF images. Two extra-focal images and one intra-focal image were taken of the full optic using the $100 \%$ mask. These were made $340 \mathrm{~mm}$ and $250 \mathrm{~mm}$ extra-focal, and $220 \mathrm{~mm}$ intra-focal from the full optic best focus position. One extra-focal image was taken of the bottom of the optic using the $48 \%$ mask. This was taken $340 \mathrm{~mm}$ extra-focal from the bottom-ofoptic best focus position.

\section{RESULTS}

The key results of the campaign are presented in this section, separated into the HEW (the key performance indicator) and the intra-focal and extra-focal images.

\subsection{HEW}

Table 4-1 presents the HEW values measured during this campaign using both single exposures and pixel scans. The pixel scan method is more precise thus it was expected that it would yield a slightly different HEW value to that of the single exposure method. Figure 4-1 shows the resulting PSF for the two pixel scan measurements.

Figure 4-2 shows the plot of all 24 azimuthal scan HEW values for the full optic. The azimuthal measurements were made prior to the pixel scans, thus it was possible to identify the region of lowest HEW - the bottom $\sim 30 \%$ of the optic. It is believed that the HEW of the optics varies in the sagittal direction due to the meridional curvature of the optics varying across the sagittal direction. Why this occurs is ongoing, but the measurements taken at cosine are compatible with this probable cause. 
Table 4-1: The HEW values of the optic for each measurement set, showing the mask used and the section of the optic imaged

\begin{tabular}{|c|c|c|c|}
\hline Mask (\%) & Section of Optic & Method & HEW (arcsec) \\
\hline 100 & Full & Single exposure & 2.7 \\
\hline 100 & Full & Pixel scan & $2.9 \pm 0.1$ \\
\hline 48 & Bottom $(\sim 30 \%)$ & Pixel scan & $2.2 \pm 0.1$ \\
\hline
\end{tabular}

(a)

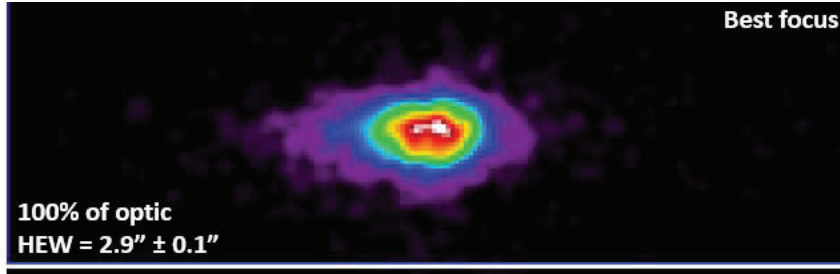

(b)

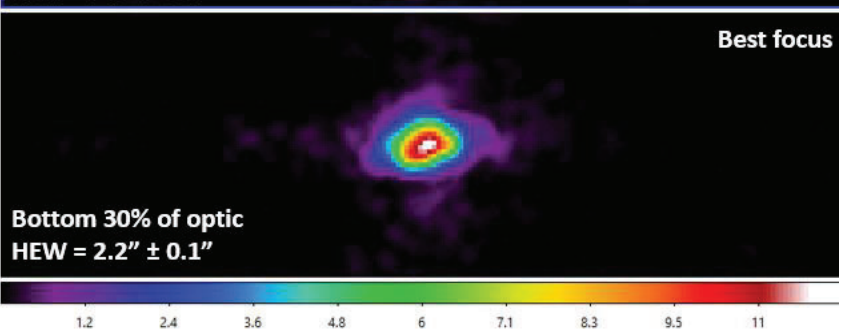

Figure 4-1: The best focus PSF image for (a) the full optic with the $100 \%$ mask, and (b) the bottom $30 \%$ of the optic with the $48 \%$ mask. The corresponding HEW values are written on the appropriate image. (Credit: MPE)

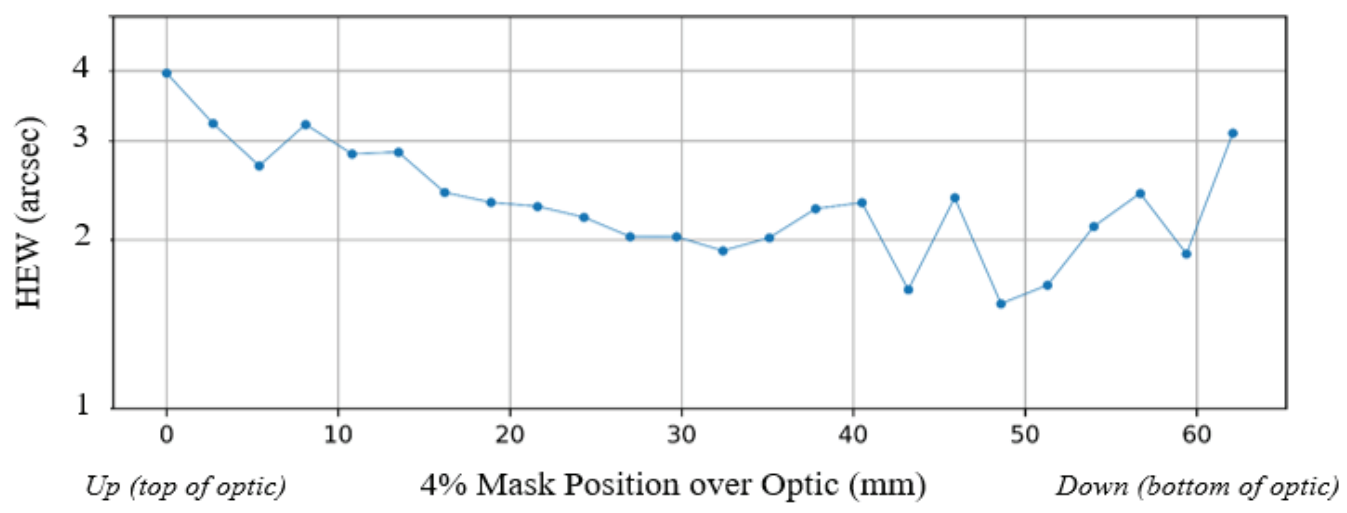

Figure 4-2: The HEW at each position of the azimuthal scan, aligning the $4 \%$ mask in front of the optic, starting at the 'top' of the optic, and measuring 24 separate positions (each one a separate exposure) to the 'bottom' of the optic. (Credit: MPE)

\subsection{Intra-Focal/Extra-Focal Images}

Figure 4-3 shows the intra-focal $(220 \mathrm{~mm})$ image for the full optic, using the $100 \%$ mask. Figure $4-4$ shows the extrafocal at $250 \mathrm{~mm}$ and Figure $4-5$ the extra-focal image at $340 \mathrm{~mm}$ for the full optic, using the $100 \%$ mask. It is clear in the distortion of the images that there is a difference across the reflecting surface of the optic. However, no unexpected features were observed in the images. 


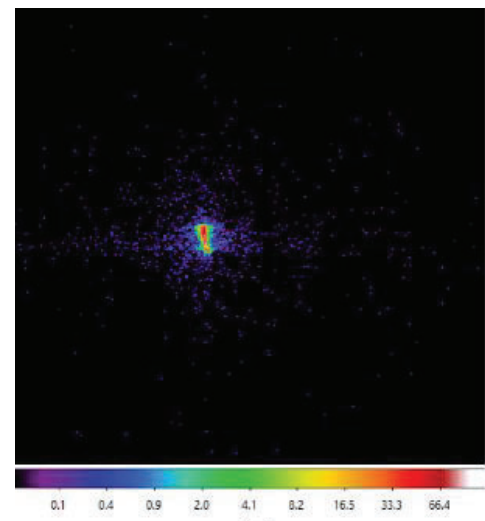

(a)

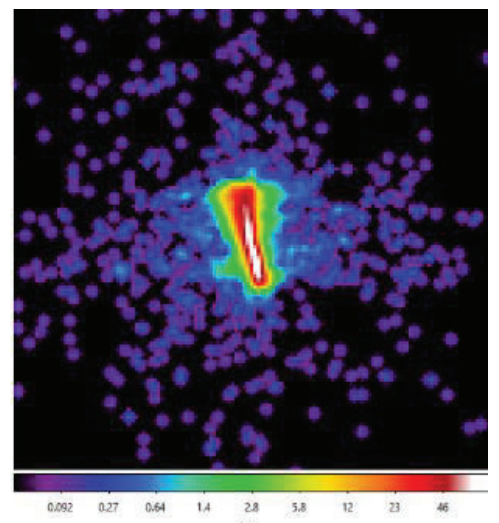

(b)

Figure 4-3: Intra-focal $220 \mathrm{~mm}$ (a) full image and (b) x4 zoomed image of XOU-0055, at the best full optic focus position, taken at Al-K using the $100 \%$ mask.

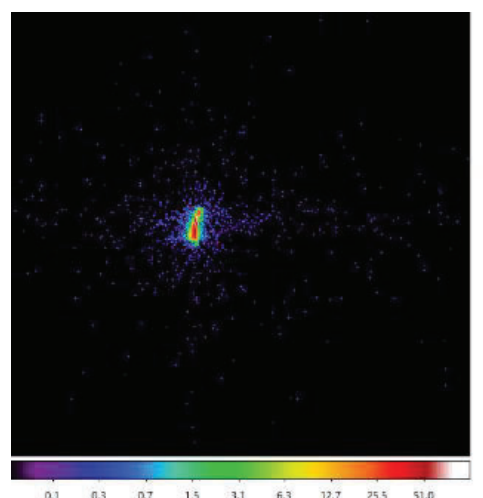

(a)

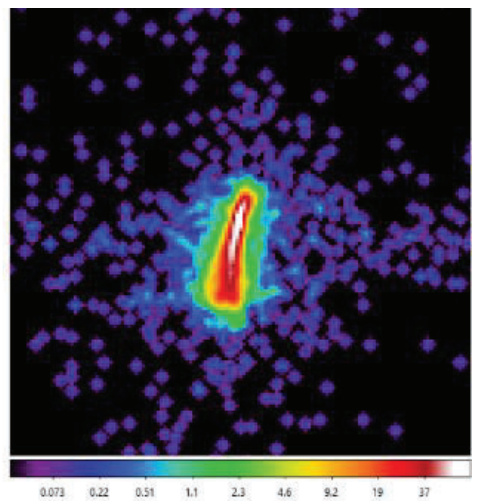

(b)

Figure 4-4: Extra-focal $250 \mathrm{~mm}$ (a) full image and (b) x4 zoomed image of XOU-0055, at the best full optic focus position, taken at Al-K using the $100 \%$ mask.

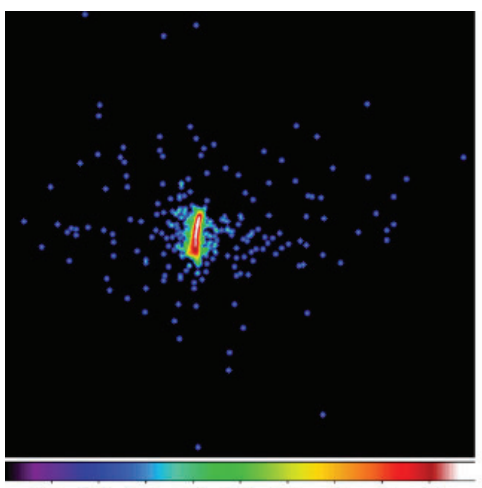

(a)

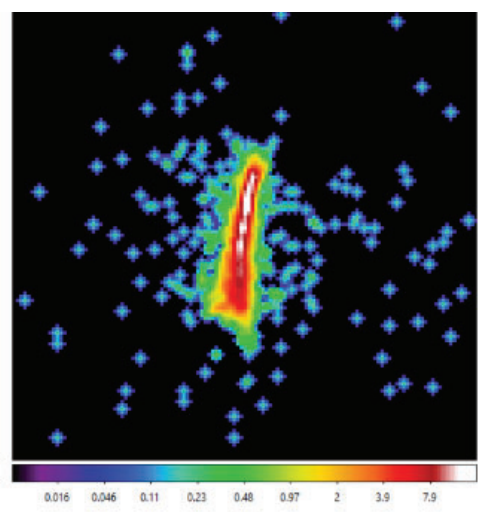

(b)

Figure 4-5: Extra-focal $340 \mathrm{~mm}$ (a) full image and (b) x4 zoomed image of XOU-0055, at the best full optic focus position, taken at Al-K using the $100 \%$ mask. 


\section{SUMMARY}

The XOU-0055 mandrel optic has a global performance of $2.9 \operatorname{arcsec} \pm 0.1 \operatorname{arcsec}(\mathrm{HEW}$ at $1.49 \mathrm{keV})$. Despite a nonsymmetric optical surface, no unexpected deviations across the surface of the optic were observed. This optic can be used to measure the performance of other x-ray integration and test facilities designed for ATHENA SPOs.

\section{ACKNOWLEDGMENTS}

Acknowledgements go to the whole PANTER team for their continuing hard work and dedication, particularly during this challenging year. MPE acknowledges the funding from the European Space Agency for testing the ATHENA optics. Part of the work performed at PANTER has been supported by the European Union Horizon 2020 Programme under the AHEAD2020 project (grant agreement n. 871158).

\section{REFERENCES}

[1] Landgraf B. et al., "Development and manufacturing of SPO X-ray mirrors", Proc. SPIE 11119, Optics for EUV, XRay, and Gamma-Ray Astronomy IX; 111190E (2019) https://doi.org/10.1117/12.2530941

[2] Collon M. et al., "Status of the silicon pore optics technology", Proceedings Volume 11119, Optics for EUV, X-Ray, and Gamma-Ray Astronomy IX; 111190L (2019) https://doi.org/10.1117/12.2530696

[3] Bradshaw M. et al., "X-Ray Testing of the Einstein Probe Follow-Up X-ray Telescope STM at MPE's PANTER Facility”, Proc. SPIE 11444, Optics for EUV, X-Ray, and Gamma-Ray Astronomy IX; 1144457 (2020) https://doi.org/10.1117/12.2560972

[4] Bradshaw M. et al., "Developments in testing x-ray optics at MPE's PANTER facility", Proc. SPIE 11119, 11119-16 (2019) https://doi.org/10.1117/12.2531709

[5] Dennerl K. et al., "Determination of the eROSITA mirror half energy width (HEW) with subpixel resolution" Proc. SPIE 8443, 844350 (2012) https://doi.org/10.1117/12.926988

[6] Burwitz V. et al., "X-ray testing at PANTER of optics for the ATHENA and Arcus Missions", Proc. SPIE 11180, 1118024 (2018) https://doi.org/10.1117/12.2535995

[7] Handick E. et al., "Upgrade of the x-ray parallel beam facility XPBF 2.0 for characterization of silicon pore optics", Proc. SPIE 11444, 114444G (2020) https://doi.org/10.1117/12.2561236 